BROOKHAVEN NATIONAL LABORATORY

Associated Universities, Inc.

Upton, L.I., N.Y.
ACCELERATOR DEPARTMENT

Interna1 Report

AADD -112

$\cos 2030.5$

II. $\$ 1.00, \operatorname{IMN}, 50$

\title{
FIELDS PRODUCED BY ELLIPTICAL CURRENT SHEETS
}

Richard A. Beth

July 6, 1966

\section{Introduction}

The vacuum chamber of a synchrotron is roughly elliptical in cross section. How should currents flowing along the elements of an elliptical cylinder be distributed to produce a desired magnetic field inside the cylinder? How should currents be distributed if a second outer chamber is provided to cancel the exterior field while producing a desired interior field?

This report gives a general solution for such problems for elliptic cylinder current sheets.

\section{Ellipses in the Complex Plane}

A convenient notation may be introduced by reviewing the following representation of ellipses in the complex plane.

Consider the analytic function of $Z=X+i Y$

$$
\Omega(z) \equiv \Gamma+i \theta=\cosh ^{-1}(z / c)=\log \left(\frac{Z+\sqrt{z^{2}-c^{2}}}{c}\right) .
$$

with branch point singularities at $z= \pm c$. Then the identification of. real and imaginary parts in

$$
z=X+i Y=c \cosh \Omega=c(\cosh \Gamma \cos \theta+i \cdot \sinh \Gamma \sin \theta)
$$

\section{LEGAL NOTICE}

This report was propared as an account of Government sponsored work. Neither the United The Commission, nor any person acting on behalf of the Commission:

A. Makes any warranty or representation, expressed or Implied, with respect to the accuA. Mampleteness, or usefuiness of the information contained in thls report, or that the use of any information, apparatus, method, or process disclosed in this report may not infringe privately owned rights; or

privately owned res or the B. Assumes any liabilitles with respect to the use of, or for datises disclosed in this report.

use of any Information, apparatus, method, or procels do commisslon" includes any emAs used in the above, "person acting on behall of the Com contractor, to the extent that ployee or contractor of the Commisston, or employee of ployee of such contractor prepares, such employee or contractor of the Connts disseminates, or provides access to, any infor mato purse 


\section{DISCLAIMER}

This report was prepared as an account of work sponsored by an agency of the United States Government. Neither the United States Government nor any agency Thereof, nor any of their employees, makes any warranty, express or implied, or assumes any legal liability or responsibility for the accuracy, completeness, or usefulness of any information, apparatus, product, or process disclosed, or represents that its use would not infringe privately owned rights. Reference herein to any specific commercial product, process, or service by trade name, trademark, manufacturer, or otherwise does not necessarily constitute or imply its endorsement, recommendation, or favoring by the United States Government or any agency thereof. The views and opinions of authors expressed herein do not necessarily state or reflect those of the United States Government or any agency thereof. 


\section{DISCLAIMER}

Portions of this document may be illegible in electronic image products. Images are produced from the best available original document. 
shows that the curves $\Gamma=$ constant, namely

$$
\cos ^{2} \theta+\sin ^{2} \theta=\left(\frac{x}{c \cosh \Gamma}\right)^{2}+\left(\frac{\dot{y}}{c \sinh \Gamma}\right)^{2}=1 \text {, }
$$

constitute a family of confocal ellipses with foci at $Z= \pm c$ and semiaxes $c \cosh \Gamma$ and $c \sinh \Gamma$. Similarly the curves $\theta=$ constant are the orthogonal family of confocal hyperbolas

$$
\cosh ^{2} \Gamma-\sinh ^{2} \Gamma=\left(\frac{X}{c \cos \theta}\right)^{2}-\left(\frac{Y}{c \sin \theta}\right)^{2}=1
$$

with the same foci.

For a fixed value of $\Gamma=\gamma=$ constant we obtain the particular ellipse

$$
z=z(\theta)=c \cosh \omega=x(\theta)+i y(\theta)
$$

where

$$
\omega=\gamma+i \theta=\lim _{z \rightarrow z(\theta)} \Omega(z)
$$

The major and minor semiaxes are, respectively,

$$
a=c \cosh \gamma, b=c \sinh \gamma \text {, }
$$

and the foci are at

$$
z= \pm c= \pm \sqrt{a^{2}-b^{2}}
$$

The real and imaginary parts of (2) give the coordinates of points on the ellipse in terms of the parameter $\theta$ :

$$
\begin{aligned}
& x(\theta)=a \cos \theta \\
& y(\theta)=b \sin \theta .
\end{aligned}
$$

\section{Elliptical Cylinder Current Sheet.}

First we evaluate the two-dimensional magnetic field produced by an elliptical cylinder current sheet which consists of current-carrying elements perpendicular to the Z-plane at the points $z(\theta)$ of the ellipse, Bq. (2). We use capital $Z$ for general field points and small $z$ for the particular $Z$ points at which currents flow. 
$\therefore$ The current sheet theorem ${ }^{*}$ requires that the complex potential, $W(Z)$, inside and outside the ellipse satisfy. :

$$
\underset{Z \rightarrow z(\theta)}{\operatorname{Lim}}\left[\mathrm{W}_{\text {out }}(Z)-\mathrm{W}_{\text {in }}(Z)\right]=4 \pi i I(\theta)+\text { constant }
$$

$I(\theta)$ is the integral of the current distribution $d I / d \theta$, where $d I$ is the upward current in the sheet interval $z(\theta)$ to $z(\theta+d \theta)$.

The most general distribution of current can be represented by a real Fourier series for $0 \leq \theta \leq 2 \pi$; we write it in the form:

$$
4 \pi \frac{d I}{d \theta}=2 I_{0}+\sum_{n=1}^{\infty} n J_{n} \cos n\left(\theta-\theta_{n}\right) .
$$

The integral is

$$
4 \pi I(\theta)=2 I_{0}\left(\theta-\theta_{0}\right)+\sum_{n=1}^{\infty} J_{n} \sin n\left(\theta-\theta_{n}\right)
$$

where $\theta_{0}$ is a constant of integration and

$$
I(2 \pi)-I(0):=I_{0}
$$

is the total current flowing in the cylinder. The reference phase, $\theta=\theta_{n}$, gives the location of a maximum of the $n^{\text {th }}$ harmonic of $d I / d \theta$, namely,

$$
z_{n}=c \cosh \omega_{n}=x_{n}+i y_{n}
$$

where

$$
\omega_{n}=\gamma+i \theta_{n}
$$

\footnotetext{
*R.A. Beth, "Fields Produced by Cylindrical Current Arrays," BNL Accelerator Dept. Internal Report AADD-102, March 25, 1966.
} 
Since, by Eq. (1), $\Omega=\Omega(Z)$ is a known function of $z$, the current sheet theorem, Eq. (3), will be satisfied if we can find a function $J(\Omega)$ such that

$$
\operatorname{Lim}_{\mathrm{Lim}} \mathrm{z}(\theta) \mathrm{J}(\Omega)=4 \pi \mathrm{iI}(\theta)
$$

and which can be separated into suitable components

$$
J(\Omega)=w_{\text {out }}(z)-\dot{w}_{\text {in }}(z)+\text { constant }
$$

to satisfy (3).

$$
\begin{aligned}
& \text { Since, from (2) and (6), } \\
& \operatorname{Lim}_{z \rightarrow z(\theta)}\left(\Omega-\omega_{n}\right)=1\left(\theta-\theta_{n}\right)
\end{aligned}
$$

and $i \sin n\left(\theta-\theta_{n}\right)=\sinh n i\left(\theta-\theta_{n}\right)$, condition (7a) is satisfied by

$$
J(\Omega)=2 I_{0} \Omega+\sum_{n=1}^{\infty} J_{n} \sinh n\left(\Omega-\omega_{n}\right)+\text { constant }
$$

with real coefficients $I_{0}$ and $J_{n}$.

Since $\Omega$, by Eq. (1), has branch points at $z= \pm c$ inside the ellipse, we assign the first term on the right, essentially

$$
2 \mathrm{I}_{0} \Omega=2 \mathrm{I}_{0} \log \left[\left(z+\sqrt{z^{2}-c^{2}}\right) \cdot c\right]
$$

to $\mathrm{W}_{\text {out }}(\mathrm{Z})$ in $(7 \mathrm{~b})$. The $\mathrm{n}^{\text {th }}$ harmonic term is $\mathrm{J}_{\mathrm{n}}$ times

$$
\sinh n\left(\Omega-\omega_{n}\right)=\frac{1}{2}\left[e^{-n \omega_{n}} e^{n \Omega}-e^{n \omega_{n}} e^{-n \Omega}\right]
$$

which, by relation (1), depends on $z$ via the $n^{\text {th }}$ powers of

$$
\begin{aligned}
& e^{\Omega}=\left(z+\sqrt{z^{2}-c^{2}}\right) / c \\
& e^{-\Omega}=c /\left(z+\sqrt{z^{2}-c^{2}}\right)=\left(z-\sqrt{z^{2}-c^{2}}\right) / c .
\end{aligned}
$$


The $e^{-n \Omega}$ term is suitable for $W_{\text {out }}(Z)$, since it is regular outside the ellipse and goes with $\mathrm{z}^{-\mathrm{n}}$ for large $z$. However, the $\mathrm{e}^{\mathrm{n} \Omega}$ term has the branch points $Z= \pm c$ and is therefore not analytic everywhere inside the ellipse as $W_{\text {in }}(Z)$ should be. The singularities can be removed by adding to (10) the identity

$$
0=\frac{1}{2}\left[e^{-n \omega_{n}} e^{-n \Omega}-e^{-n \omega_{n}} e^{-n \Omega}\right]
$$

to obtain

$$
\sinh n\left(\Omega-\omega_{n}\right)=e^{-n \omega_{n}} \cosh n \Omega-\cosh n \omega_{n} e^{-n \Omega}
$$

.where

$$
\cosh \mathrm{n} \Omega=\mathrm{T}_{\mathrm{n}}(Z / \mathrm{c})
$$

is a Chebyshev ${ }^{*}$ polynomial of degree $n$ in $(z / c)$. This polynomial is obviously regular everywhere within the ellipse.

Hence we identify the parts of (8) in (7b):

$$
\begin{aligned}
& W_{\text {out }}(Z)=2 I_{0} \Omega-\sum_{n=1}^{\infty} J_{n} \cosh n \omega_{n} e^{-n \Omega}+\text { constant } \\
& W_{\text {in }}(Z)=\sum_{n=1}^{\infty} J_{n} e^{-n \omega_{n} \cosh n \Omega+\text { constant }}
\end{aligned}
$$

to satisfy the current sheet theorem for the current distribution (4).

* Though the transliteration "Tschebyscheff" is common, we follow the more convenient National Bureau of Standards spelling in "Handbook of Mathematical Functions," edited by M. Abramowitz and I.A. Stegun (U.S. Government Printing Office, Washington, D.C., 1964), p. 795. 
Since $z=c \cosh \Omega$ and $z_{n}=c \cosh \omega_{n}=a \cos \theta_{n}+i b \sin \theta_{n}$ we can write (13) as explicit functions of $z$ in the form

$$
\begin{aligned}
& W_{\text {out }}(z)=2 I_{0} \log \left(z+\sqrt{z^{2}-c^{2}}\right)-\sum_{n=1}^{\infty} J_{n} \frac{c^{n} T_{n}\left(\frac{z_{n}}{c}\right)}{\left(z+\sqrt{z^{2}-c^{2}}\right)^{n}}+\text { const. } \\
& W_{i n}(z)= \\
& \quad-\sum_{n=1}^{\infty} J_{n} \frac{c^{n} T_{n}\left(\frac{z}{c}\right)}{(a+b)^{n} e^{i n} n}+\text { const. }
\end{aligned}
$$

where the Chebyshev polynomial numerators are

$$
\begin{aligned}
& c^{n} T_{n}\left(\frac{z}{c}\right)=\frac{1}{2}\left[(a+b)^{n} e^{i n \theta_{n}}+(a-b)^{n} e^{-i n \theta_{n}}\right] \\
& c^{n} T_{n}\left(\frac{z}{c}\right)=\frac{1}{2}\left[\left(z+\sqrt{z^{2}-c^{2}}\right)^{n}+\left(z-\sqrt{z^{2}-c^{2}}\right)^{n}\right] .
\end{aligned}
$$

In the complex representation both components of magnetic field are obtained in the form

$$
\mathrm{H}_{\mathrm{Y}}+\mathrm{iH} \mathrm{H}_{\mathrm{X}}=\mathrm{H}(\mathrm{Z})=\frac{\mathrm{dW}}{\mathrm{dz}}
$$

by differentiating the complex potential $W(Z)$. Thus the fields due to the general elliptical current sheet are

$$
\begin{aligned}
& H_{\text {out }}(Z)=\left[2 I_{0}+\sum_{n=1}^{\infty} n J_{n} \cosh n \omega_{n} e^{-n \Omega}\right] / c \sinh \Omega \\
& H_{\text {in }}(Z)=\left[-\sum_{n=1}^{\infty} n J_{n} e^{-n \omega_{n}} \sinh n \Omega\right] / c \sinh \Omega
\end{aligned}
$$

since

$$
\frac{d \Omega}{d z}=\frac{1}{c \sinh \Omega} \text {. }
$$


We note that $c \sinh \Omega=\sqrt{z^{2}-c^{2}}$ and that

$$
\frac{\sinh n \Omega}{\sinh \Omega}=U_{n-1}\left(\frac{z}{c}\right)
$$

is a Chebyshev polynomial of the second kind ${ }^{*}$ of degree $n-1$ in $(z / c)$.

\section{Current Distribution to Produce a Given Field}

Equations (13) solve the problem of finding the field produced by an arbitrary current distribution (4) on an elliptic cylinder. We can now also solve the inverse problem of finding the current distribution needed to produce an arbitrarily given field inside an elliptic cylinder.

We choose the $X$ and $Y$ axes of the $Z$ plane in the direction of the major and minor semiaxes, $a>b$, of the ellipse and let $Z=0$ be the center of the ellipse. The most general nonsingular field within the ellipse has an analytic potential $W_{\text {in }}(Z)$ expressible as a series of positive powers of $(z / c)$, where $c^{2}=a^{2}-b^{2}$. Any power $(z / c)^{m} c a n$, in turn, be expressed as a linear combination of Chebyshev polynomials $T_{n}(z / c)$ with $0 \leq n \leq m$. ** Hence the most general internal potential can be written as a series of Chebyshev polynomials with complex coefficients:

$$
W_{i n}(Z)=\sum_{n=1}^{\infty}\left(A_{n}+i B_{n}\right) T_{n}(Z / c)+\text { constant. }
$$

Identification with (14b) shows that we:must have a current distribution (4) with

$$
J_{n}=-\left(\frac{a+b}{c}\right)^{n} \sqrt{A_{n}^{2}+B_{n}^{2}}
$$

and.

$$
\tan n \theta_{n}=-B_{n} / A_{n}
$$

\footnotetext{
* See NBS "Handbook of Mathematical Functions," p. 796. ${ }^{* *}$ Ibid., p. 795 .
} 


\section{Two Elliptic Cylinders with Zero Externa1 Field}

The form of the $Z$ dependence of $W_{\text {out }}(Z)$ in (13) suggests that a pair of confocal elliptical current sheets can be arranged to cancel the external field while producing an arbitrarily prescribed nonsingular field, (18),

$$
w_{i n}(Z)=\sum_{n=1}^{\infty}\left(A_{n}+i B_{n}\right) \cosh n+\text { constant }
$$

within the inner cylinder.

While the equations can be found by superposition of the single cylinder results it is simpler to apply the current sheet theorem directly.

We use the same notation as before but denote quantities relating to the outer cylinder by primes. The complex potentials inside, between, and outside the two cylinders will be related by appropriate $J(\Omega)$ functions as defined in (7):

$$
\begin{aligned}
& \mathrm{J}(\Omega)=\mathrm{w}_{\text {betw }}-\mathrm{w}_{\text {in }} \\
& \mathrm{J}^{\prime}(\Omega)=\mathrm{w}_{\text {out }}-\mathrm{w}_{\text {betw }} .
\end{aligned}
$$

Hence, when we set $\mathrm{W}_{\text {out }}=0$ and use the form $(8)$ for $\mathrm{J}(\Omega)$ and $\mathrm{J}^{\prime}(\Omega)$, we. obtain

$W_{\text {in }}=-J-J^{\prime}=-2\left(I_{0}+I_{0}^{\prime}\right) \Omega-\sum_{n=1}^{\infty}\left[J_{n} \sinh n\left(\Omega-w_{n}\right)+J_{n}^{\prime} \sinh n\left(\Omega-w_{n}^{\prime}\right)\right]+\operatorname{const}$.

$\mathrm{W}_{\text {betw }}=-J^{\prime}=-2 I_{0}^{\prime} \Omega-\sum_{n=1}^{\infty} J_{n}^{\prime} \sinh n\left(\Omega-w_{n}^{\prime}\right)+$ const.

where $\omega_{n}=\gamma+i \theta_{n}$ and $\omega_{n}^{\prime}=\gamma^{\prime}+i \theta_{n}^{\prime}$.

With $W_{\text {in }}$ prescribed by (20) we wish to evaluate $W_{\text {betw }}$ and the current distributions $d I / d \theta$ and $d^{\prime} / d \theta$ in the form (4) for given elliptic cylinders, i.e., for given values of $\gamma$ and $\gamma^{\prime}$. Specifically, we need to evaluate 


$$
\begin{gathered}
4 \pi \frac{d I}{d \theta}=2 I_{0}+\sum_{n=1}^{\infty} n J_{n}\left(\cos n \theta_{n} \cos \theta+\sin n \theta_{n} \sin \theta\right) \\
4 \pi \frac{d I^{\prime}}{d \theta}=2 I_{0}^{\prime}+\sum_{n=1}^{\infty} n J_{n}^{\prime}\left(\cos n \theta_{n}^{\prime} \cos \theta+\sin n \theta_{n}^{\prime} \sin \theta\right)
\end{gathered}
$$

and

$$
W_{\text {betw }}=-2 I_{0}^{\prime} \Omega-\sum_{n=1}^{\infty} J_{n}^{\prime}\left[\cos n \theta_{n}^{\prime} \sinh n\left(\Omega-\gamma^{\prime}\right)-i \sin n \theta_{n}^{\prime} \cosh n\left(\Omega-\gamma^{\prime}\right)\right] \text {. }
$$

Thus the problem is reduced to finding the real coefficients

$$
\begin{array}{ll}
J_{n} \cos n \theta_{n} & J_{n} \sin n \theta_{n} \\
J_{n}^{\prime} \cos n \theta_{n}^{\prime} & J_{n}^{\prime} \sin n \theta_{n}^{\prime}
\end{array}
$$

in terms of the given real parameters

$$
A_{n}, B_{n}, \gamma, \text { and } Y^{\prime} \cdot
$$

If we equate harmonics in (20) and (21) we obtain $I_{0}+I_{0}^{\prime}=0$ [hence arbitrary $I_{o}^{\prime}=-I_{0}$ does not affect (20)], and

$$
J_{n} \sinh n\left(\Omega-\omega_{n}\right)+J_{n}^{\prime} \sinh n\left(\Omega-\omega_{n}^{\prime}\right)=-\left(A_{n}+i B_{n}\right) \cosh n \Omega .
$$

The latter holds for all $\Omega$; hence

$$
\begin{aligned}
& J_{n} \cosh n \omega_{n}+J_{n}^{\prime} \cosh n \omega_{n}^{\prime}=0 \\
& J_{n} \sinh n \omega_{n}+J_{n}^{\prime} \sinh n \omega_{n}=A_{n}+i B_{n} .
\end{aligned}
$$

By equating real and imaginary parts we find, since $J_{n}$ and $J_{n}^{\prime}$ are real:

$$
\begin{array}{ll}
J_{n} \cos n \theta_{n}=-\frac{A_{n}}{\beta_{n}} \cosh n \gamma^{\prime} & J_{n} \sin n \theta_{n}^{\prime}=\frac{B_{n}}{\beta_{n}} \sinh \gamma^{\prime} \\
J_{n}^{\prime} \cos n \theta_{n}^{\prime}=\frac{A_{n}}{\beta_{n}} \cosh \gamma \gamma & J_{n}^{\prime} \sin n \theta_{n}^{\prime}=-\frac{B_{n}}{\beta_{n}} \sinh \gamma^{\prime} \gamma
\end{array}
$$

where

$$
\beta_{n}=\sinh n\left(\gamma^{\prime}-\gamma\right)
$$


With these values for the coefficients, Equations (23) and (24) give the general solution of our two cylinder problem with zero outside field.

Both magnetic field components between the cylinders can be obtained by differentiating (24) with respect to $Z$ since $d W / d Z=H_{Y}+i H_{X}$ in this complex representation.

It is useful to express the functions of $\gamma$ and $\gamma^{\prime}$.in (25) in terms of the semiaxes of the confocal ellipses:

$$
\begin{array}{ll}
a=c \cosh \gamma & \mathbf{a}^{\prime}=c \cosh \gamma^{\prime} . \\
\mathbf{b}=c \sinh \gamma & \mathbf{b}^{\prime}=c \sinh \gamma^{\prime} .
\end{array}
$$

Adding and subtracting yields

$$
e^{\gamma}=\frac{a+b}{c}=\frac{c}{a-b} \quad e^{\gamma^{\prime}}=\frac{a^{\prime}+b^{\prime}}{c}=\frac{c}{a^{\prime}-b}
$$

whence

$$
\begin{aligned}
& \cosh n Y=\left[(a+b)^{n}+(a-b)^{n}\right] / 2 c^{n} \\
& \sinh n Y=\left[(a+b)^{n}-(a-b)^{n}\right] / 2 c^{n}
\end{aligned}
$$

and similar expressions for the primed quantities.

It is further.useful to introduce

$$
p=\frac{a^{\prime}+b^{\prime}}{a+b}=\frac{a-b}{a^{\prime}-b^{\prime}}=e^{\gamma^{\prime}-\gamma}
$$

as a measure of the ratio of the sizes of the two ellipses; then

$$
\beta_{n}=\sinh n\left(\gamma^{\prime}-\gamma\right)=\frac{1}{2}\left(\rho^{n}-\rho^{-n}\right)
$$

By using the relations (26) and (27) the coefficients (25) can be computed algebraically from the semiaxes of the ellipses and the given $A_{n}$ and $B_{n}$. 


\section{Conclusion}

Special cases illustrating these general solutions have been given in previous reports.

The current distributions required on elliptic cylinders to produce an internal constant gradient field are given in AADD-110 together with the other field produced in the one and two cylinder cases.

For $b=a$ the elliptical solutions reduce to those for circular cylinders. The single circular cylinder is treated in AADD-102 and the two cylinder case with zero outside field in AADD-103.

Finally it should be noted that, for both elliptic and circular cases; the two cylinder results reduce to the single cylinder expressions when the outer cylinder is made infinitely large.
$\mathrm{RAB} /$ yew
$7 / 14 / 66$
Distr.: AADD External 